\title{
A GENERAL VECTOR-VALUED VARIATIONAL INEQUALITY AND ITS FUZZY EXTENSION
}

\author{
SEHIE PARK \\ Department of Mathematics \\ Seoul National University \\ Seoul 151-742. KOREA \\ BYUNG-SOO LEE \\ Department of Mathematics \\ Kyungsung University \\ Pusan 608-736. KOREA \\ GUE MYUNG LEE \\ Department of Applied Mathematics \\ Pukyong National University \\ Pusan 608-737, KOREA
}

(Received December 12, 1996)

\begin{abstract}
A general vector-valued variational inequality (GVVI) is considered. We establish the existence theorem for (GVVI) in the noncompact setting, which is a noncompact generalization of the existence theorem for (GVVI) obtained by Lee et al., by using the generalized form of KKM theorem due to Park. Moreover, we obtain the fuzzy extension of our existence theorem.
\end{abstract}

KEY WORDS AND PHRASES: Variational inequalities, fuzzy extension, KKM theorem. 1991 AMS SUBJECT CLASSIFICATION CODES: $47 \mathrm{H} 19$.

\section{INTRODUCTION}

Recently, Giannessi [1] introduced a variational inequality for vector-valued mappings in a Euclidean space. Since then, Chen et al. [2-6] have intensively studied variational inequalities for vector-valued mappings in Banach spaces. Lee et al. [7] have established the existence theorem of a variational inequality for a multifunction with vector values in a Banach space.

On the other hand, Chang and Zhu [8] introduced the concept of variational inequalities for fuzzy mappings in locally convex Hausdorff topological vector spaces and investigated existence theorems for some kinds of variational inequalities for fuzzy mappings, which were the fuzzy extensions of some theorems in $[9,10,11,12]$. Lee et al. [13] obtained the fuzzy generalizations of new results of Kim and Tan [14], and they [7] established the fuzzy extension of their existence theorem. Our motivation of this paper is to consider the noncompact cases of the existence theorems of variational inequalities for multifunctions with vector values or fuzzy mappings in Banach spaces obtained by Lee et al. [7]

Let $X$ and $Y$ be two normed spaces and $D$ a nonempty convex subset of $X$. Let $T: X \rightarrow 2^{L(X, Y)}$ be a multifunction, where $L(X, Y)$ is the space of all continuous linear maps from $X$ into $Y$, and $C$ a closed pointed and convex cone of $Y$ such that Int $C \neq \emptyset$, where Int denotes the interior.

Consider the following generalized vector-valued variational inequality:

(GVVI) Find $x_{0} \in D$ such that for each $x \in D$, there exists an $s_{0} \in T\left(x_{0}\right)$ such that

$$
\left\langle s_{0}, x-x_{0}\right\rangle \notin-I n t C,
$$

where $\left\langle s_{0}, y\right\rangle$ denotes the evaluation of $s_{0}$ at $y$. 
When $T$ is a mapping from $X$ into $L(X, Y)$, (GVVI) reduces to the following vector-valued variational inequality (VVI) considered by Chen et al. $[3,5,6]$.

(VVI) Find $x_{0} \in D$ such that $\left\langle T\left(x_{0}\right), x-x_{0}\right\rangle \notin-I n t C$ for all $x \in D$.

The above inequality (VVI) is a generalization of the following classic scalar-valued variational inequality (VI).

(VI) Find $x_{0} \in D$ such that $\left\langle f\left(x_{0}\right), x-x_{0}\right\rangle \geq 0$ for all $x \in D$, where $f: \mathbb{R}^{n} \rightarrow \mathbb{R}^{n}$ is a given mapping.

Our purpose in this paper is to establish the existence theorems for (GVVI) in the noncompact setting, which is the noncompact case of the existence theorem for (GVVI) obtained by Lee et al. [7], by using a particular form of the generalized KKM theorems due to Park [15-17]. Our existence theorem subsumes Theorem 2.1 of Cottle and Yao [18], the part (i) of Theorem 2.1 of Chen and Yang [6], Theorem 2 of Yang [19] and Theorem 2.1 of Lee et al. [7]. Moreover, we obtain the fuzzy extension of our existence theorem. Our fuzzy extension is a generalization of Theorem 3.1 of Lee et al. [7]. Now we give the definition of a KKM map.

DEFINITION 1.1. Let $D$ be a subset of a convex space $X$. Then a multifunction $G: D \rightarrow 2^{X}$ is called KKM if for each nonempty finite subset $N$ of $D, \operatorname{co} N \subset G(N)$, where co denotes the convex hull and $G(N)=\bigcup\{G x: x \in N\}$.

A convex space $X$ is a nonempty convex set (in a vector space) with any topology that induces the Euclidean topology on the convex hulls of its finite subsets. Thus, a convex subset $X$ of a topological vector space $E$ with the relative topology is automatically a convex space. For details of the convex space, see Lassonde [9].

We say that a subset $A$ of a topological space $X$ is compactly closed in $X$ if for every compact subset $K \subset X$ the set $A \cap K$ is closed in $K$. We need the following particular form of the generalized KKM theorems due to Park [16-18], which will be used in the proof of our Theorem 2.

THEOREM 1. Let $X$ be a convex space, $K$ a nonempty compact subset of $X$, and $G: X \rightarrow 2^{X}$ a KKM multifunction. Suppose that

(1) for each $y \in X, G(y)$ is compactly closed; and

(2) for each finite subset $N$ of $X$, there exists a compact convex subset $L_{N}$ of $X$ such that $N \subset L_{N}$ and $L_{N} \cap \bigcap\left\{G(y): y \in L_{N}\right\} \subset K$.

Then we have

$$
K \cap \bigcap\{G(y): y \in X\} \neq \emptyset
$$

\section{Existence Theorems}

First, we give the following definitions for the existence theorems for (GVVI).

DEFINITION 2.1. Let $X$ be a normed space with dual space $X^{*}$ and $T: X \rightarrow X^{*}$ a mapping.

1. $T$ is said to be monotone if for any $x, y \in X,\langle T(x)-T(y), x-y\rangle \geq 0$.

2. $T$ is said to be pseudomonotone if for any $x, y \in X,\langle T(x), y-x\rangle \geq 0$ implies that $\langle T(y), y-x\rangle \geq 0$.

3. $T$ is said to be hemicontinuous if for any $x, y, z \in X$, the mapping $\alpha \mapsto\langle T(x+\alpha y), z\rangle$ is continuous at $0^{+}$.

DEFINITION 2.2. Let $X, Y$ be two normed spaces, $T: X \rightarrow L(X, Y)$ a mapping and $C$ a closed, pointed and convex cone of $Y$ such that $\operatorname{Int} C \neq \emptyset$.

1. $T$ is said to be $C$-monotone if for any $x, y \in X,\langle T(x)-T(y), x-y\rangle \in C$.

2. $T$ is said to be $C$-pseudomonotone if for any $x, y \in X,\langle T(x), y-x\rangle \notin-I n t C$ implies that $\langle T(y), y-x\rangle \notin-$ Int $C$. 
3. $T$ is said to be $V$-hemicontimuous if for any $x, y, z \in X$, the mapping $\alpha \mapsto\langle T(x+\alpha y), z\rangle$ is continuous at $0^{+}$.

REMARK. When $Y=\mathbb{R}$ and $C=\mathbb{R}_{+}$, Definition 2.2 becomes Definition 2.1 .

DEFINITION 2.3. Let $X$ and $Y$ be two normed spaces, $T: X \rightarrow 2^{L(X, Y)}$ a set-valued map and $C$ a closed, pointed and convex cone of $Y$ such that Int $C \neq \emptyset$.

1. $T$ is said to be $C$-monotone if for any $x, y \in X, s \in T(x)$ and $t \in T(y),\langle s-t, x-y\rangle \in C$

2. $T$ is said to be $C$-pseudomonotone if for any $x, y \in X,\langle s, y-x\rangle \notin-\operatorname{Int} C$ for some $s \in T(x)$ implies that $\langle t, y-x\rangle \notin-I n t C$ for some $t \in T(y)$.

3. $T$ is said to be $V$-hemicontinuous if for any $x, y \in X, \alpha>0$ and $t_{\alpha} \in T(x+\alpha y)$, there exists $t_{0} \in T(x)$ such that for any $z \in X,\left\langle t_{\alpha}, z\right\rangle \mapsto\left\langle t_{0}, z\right\rangle$ as $\alpha \rightarrow 0^{+}$.

REMARK. 1. Definition 2.3 is a generalization of Definition 2.2 .

2. We can easily prove that the $C$-monotonicity implies the $C$-pseudomonotonicity.

Now we prove the following existence theorem for the noncompact case of (GVVI).

THEOREM 2. Let $X$ and $Y$ be Banach spaces, $C$ a closed, pointed and convex cone in $Y$ with Int $C \neq \emptyset, D$ a nonempty convex subset of $X, K$ a nonempty compact subset of $X$, and $T: X \rightarrow 2^{L(X, Y)}$. Suppose that

(1) $T$ is $C$-pseudomonotone, compact-valued, and $V$-hemicontinuous; and

(2) for each nonempty finite subset $N$ of $D$, there exists a nonempty compact convex subset $L_{N}$ of $D$ such that $N \subset L_{N}$ and for each $x \in L_{N} \backslash K$ there exists a $y \in L_{N}$ such that $\langle t, y-x\rangle \in-\operatorname{Int} C$ for all $t \in T(y)$. Then (GVVI) is solvable.

PROOF. Define a multifunction $F_{1}: D \rightarrow 2^{D}$ by

$$
F_{1}(y)=\{x \in D:\langle s, y-x\rangle \notin-\operatorname{Int} C \text { for some } s \in T(x)\}
$$

for $y \in D$. Then $F_{1}$ is a $\mathrm{KKM}$ multifunction on $D$.

In fact, suppose that $N=\left\{x_{1}, \cdots, x_{n}\right\} \subset D, \sum_{i=1}^{n} \alpha_{i}=1, \alpha_{i} \geq 0, i=1, \cdots, n \quad$ and $x=\sum_{i=1}^{n} \alpha_{\imath} x_{i} \notin F_{1}(N)$. Then for any $s \in T(x)$, we have $\left\langle s, x_{i}-x\right\rangle \in-I n t C, i=1, \cdots, n$ Thus we have

$$
\langle s, x\rangle=\left\langle s, \sum_{i=1}^{n} \alpha_{i} x_{i}\right\rangle=\sum_{i=1}^{n} \alpha_{i}\left\langle s, x_{i}\right\rangle \in \sum_{i=1}^{n} \alpha_{i}\langle s, x\rangle-\operatorname{Int} C=\langle s, x\rangle-\text { int } C .
$$

Hence $0 \in \operatorname{Int} C$, which contradicts the pointedness of $C$. Therefore, $F_{1}$ is a KKM multifunction on $D$

Define a multifunction $F_{2}: D \rightarrow 2^{D}$ by

$$
F_{2}(y)=\{x \in D:\langle t, y-x\rangle \notin-I n t C \text { for some } t \in T(y)\}
$$

for $y \in D$. For any $x \in F_{1}(y)$ there exists an $s \in T(x)$ such that $\langle s, y-x\rangle \notin-I n t C$. By the $C$ pseudomonotonicity of $T$, there exists a $t \in T(y)$ such that $\langle t, y-x\rangle \notin-\operatorname{Int} C$. Thus $x \in F_{2}(y)$ Hence for any $y \in D, F_{1}(y) \subset F_{2}(y)$. Therefore $F_{2}$ is also a KKM multifunction on $D$.

We claim that $F_{2}$ is closed-valued. In fact, for any $y \in D$, let $\left\{x_{n}\right\}$ be a sequence in $F_{2}(y)$ which converges to $x_{*} \in D$. Since $x_{n} \in F_{2}(y)$ for each $n$, there exists a $t_{n} \in T(y)$ such that $\left\langle t_{n}, y-x_{n}\right\rangle \in Y \backslash(-I n t C)$. Since $T(y)$ is compact, we may assume that $\left\{t_{n}\right\}$ converges to some $t_{*} \in T(y)$. Note that

$$
\begin{aligned}
\left\|\left\langle t_{n}, y-x_{n}\right\rangle-\left\langle t_{*}, y-x_{*}\right\rangle\right\| & =\left\|\left\langle t_{n}, x_{*}-x_{n}\right\rangle-\left\langle t_{n}-t_{*}, y-x_{*}\right\rangle\right\| \\
& \leq\left\|\left\langle t_{n}, x_{*}-x_{n}\right\rangle\right\|+\left\|\left\langle t_{n}-t_{*}, y-x_{*}\right\rangle\right\| \\
& \leq\left\|t_{n}\right\|\left\|x_{*}-x_{n}\right\|+\left\|t_{n}-t_{*}\right\|\left\|y-x_{*}\right\| .
\end{aligned}
$$

Since $\left\{t_{n}\right\}$ is bounded in $L(X, Y),\left\langle t_{n}, y-x_{n}\right\rangle$ converges to $\left\langle t_{*}, y-x_{*}\right\rangle$. Hence $\left\langle t_{*}, y-x_{*}\right\rangle \notin-\operatorname{Int} C$, whence we have $x_{*} \in F_{2}(y)$. 
Further, note that assumption (2) implies that, for each $x \in L_{N} \backslash K$ there exists a $y \in L_{N}$ such that $x \notin F_{2}(y)$. Hence $L_{N} \cap \bigcap\left\{F_{2}(y): y \in L_{N}\right\} \subset K$. Therefore, condition (2) of Theorem 1 holds.

Therefore, by Theorem 1, there exists an $x \in K \cap \bigcap\left\{F_{2}(y): y \in D\right\}$. Then for any $y \in D$, there exists a $t_{y} \in T y$ such that $\left\langle t_{y}, y-x\right\rangle \notin-I n t C$. By the convexity of $D$, for any $\alpha \in(0,1)$, there exists a $t_{\alpha} \in T(\alpha y+(1-\alpha) x)$ such that $\left\langle t_{\alpha}, \alpha(y-x)\right\rangle \notin-I n t C$. Dividing by $\alpha$, we have $\left\langle t_{\alpha}, y-x\right\rangle \notin-I n t C$. By the $V$-hemicontinuity of $T$, there exists $t_{0} \in T(x)$ such that $\left\langle t_{0}, y-x\right\rangle \notin-I n t C$. Hence $x \in \bigcap\left\{F_{1}(y): y \in D\right\} \neq \emptyset$. Consequently, there exists an $x_{0} \in K$ such that for each $x \in D$, there exists an $s_{0} \in T\left(x_{0}\right)$ such that $\left\langle s_{0}, x-x_{0}\right\rangle \notin-I n t C$.

COROLLARY 2.1. In Theorem 2 , if $D$ is closed, then the coercivity (2) can be replaced by the following without affecting its conclusion:

(2') there exists a nonempty compact subset $K$ of $D$ and a $y_{0} \in K$ such that

$$
\left\langle t, y_{0}-x\right\rangle \in-\text { Int } C \text { for } x \in D-K \text { and } t \in T\left(y_{0}\right) \text {. }
$$

PROOF. It suffices to show that (2') implies (2). In fact, for any nonempty finite subset $N$ of $D$, we let $L_{N}=\bar{c} o\left(\left\{y_{0}\right\} \cup N \cup K\right) \subset D$. By (2'), for any $x \in L_{N}-K \subset D-K$, there exists a $y_{0} \in K \subset L_{N}$ such that $\left\langle t, y_{0}-x\right\rangle \in-\operatorname{Int} C$ for all $t \in T\left(y_{0}\right)$. Hence (2) holds.

REMARK. Even for a single-valued $T$, Corollary 2.1 is more general than Yang [19, Theorem 2].

For $D=K$, Theorem 2 reduces to the following

COROLLARY 2.2 Let $X$ and $Y$ be Banach spaces, $C$ a closed pointed and convex cone in $Y$ with Int $C \neq \emptyset, D$ a nonempty compact and convex subset of $X$ and $T: X \rightarrow 2^{L(X, Y)} C$-pseudomonotone, compact-valued, and $V$-hemicontinuous. Then (GVVI) is solvable.

REMARK. Corollary 2.2 extends Chen and Yang [6, Theorem 2.1, Part (i)].

COROLLARY 2.3 [7]. Let $X$ be a reflexive Banach space, $Y$ a Banach space, $C$ a closed pointed and convex cone in $Y$ with Int $C \neq \emptyset, D$ a nonempty bounded closed and convex subset of $X$, and $T: X \rightarrow 2^{L(X, Y)} C$-pseudomonotone, compact-valued and $V$-hemicontinuous. Then (GVVI) is solvable.

PROOF. Switch to the weak topology on $X$.

COROLLARY 2.4. Let $X$ be a Banach space with dual space $X^{*}, D$ a nonempty compact and convex subset of $X$ and $T: X \rightarrow X^{*}$ pseudomonotone and hemicontinuous. Then there exists an $x_{0} \in D$ such that $\left\langle T\left(x_{0}\right), x-x_{0}\right\rangle \geq 0$ for all $x \in D$.

REMARK. Corollary 2.4 generalizes Cottle and Yao [11, Theorem 2.1]. Note that for $Y=\mathbb{R}$ and $C=\mathbf{R}_{+}$, corollaries extend or reduce to well-known scalar valued variational inequalities due to Hartman and Stampacchia, Browder, Stampacchia, Mosco, Dungundji and Granas and many others.

\section{FUZZY EXTENSION}

Let $X$ and $Y$ be two normed spaces and $\mathcal{F}(L(X, Y))$ the collection of all fuzzy sets on $L(X, Y)$. A mapping $F$ from $X$ into $\mathcal{F}(L(X, Y))$ is called a fuzzy mapping.

If $F: X \rightarrow \mathcal{F}(L(X, Y))$ is a fuzzy mapping, then $F(x), x \in X$ (denoted by $F_{x}$ ), is a fuzzy set in $\mathcal{F}(L(X, Y))$ and $F_{x}(s), s \in L(X, Y)$, is the degree of membership of $s$ in $F_{x}$. Let $A \in \mathcal{F}(L(X, Y))$ and $\beta \in[0,1]$. Then the set $(A)_{\beta}=\{s \in L(X, Y): A(s) \geq \beta\}$ is said to be an $\alpha$-cut set of $A$.

DEFINITION 3.1 [20]. A fuzzy set $A$ in $L(X, Y)$ is compact if for each $\beta \in(0,1],(A)_{\beta}$ is compact in $L(X, Y)$.

DEFINITION 3.2. Let $X$ and $Y$ be two normed spaces, $F: X \rightarrow \mathcal{F}(L(X, Y))$ a fuzzy mapping and $C$ a closed, pointed and convex cone of $Y$ such that Int $C \neq \emptyset$.

1. $F$ is said to be $C$-monotone if for any $x, y \in X$ and $s, t \in L(X, Y)$ with $F_{x}(s)>0$ and $F_{y}(t)>0,\langle s-t, x-y\rangle \in C$. 
2. $F$ is said to be $C$-pseudomonotone if for any $x, y \in X$ and $\beta \in(0,1],\langle s, y-x\rangle \notin-I$ int $C$ for some $s \in L(X, Y)$ with $F_{x}(s) \geq \beta$ implies that $\langle t, y-x\rangle \notin-I n t C$ for some $t \in L(X, Y)$ with $F_{y}(t)>\beta$.

3. $F$ is said to be hemicontimuous if for any $x, y \in X$ and $t_{\alpha} \in L(X, Y)$ with $F_{x+\alpha y}(t \alpha) \geq \beta$ where $\beta \in(0,1]$, there exists $t_{0} \in L(X, Y)$ with $F_{x}\left(t_{0}\right) \geq \beta$ for any $z \in X,\left\langle t_{\alpha}, z\right\rangle \rightarrow\left\langle t_{0}, z\right\rangle$ as $\alpha \rightarrow 0^{+}$.

Now we obtain a fuzzy extension of Theorem 2.

THEOREM 3. Let $X$ and $Y$ be Banach spaces, $C$ a closed, pointed and convex cone in $Y$ with Int $C \neq \emptyset, D$ a nonempty convex subset of $X, K$ a nonempty compact subset of $X$, and $F: X \rightarrow \mathcal{F}(L(X, Y))$ a fuzzy mapping such that there exists a real number $\beta \in(0,1]$ such that for each $x \in X,\left(F_{x}\right)_{\beta}$ is a nonempty subset of $L(X, Y)$. Suppose that

(1) $F$ is $C$-pseudomonotone and hemicontinuous, and for each $x \in X, F_{x}$ is a compact fuzzy set in $L(X, Y)$,

(2) for each nonempty finite subset $N$ of $D$, there exists a compact convex subset $L_{N}$ of $D$ such that $N \subset L_{N}$ and for each $x \in L_{N} \backslash K$ there exists a $y \in L_{N}$ such that $\langle t, y-x\rangle \in-$ Int $C$ for all $t \in L(X, Y)$ with $F_{y}(t) \geq \beta$.

Then there exists an $x_{0} \in D$ such that for each $x \in D$, there exists an $s_{0} \in L(X, Y)$ with $F_{x_{0}}\left(s_{0}\right) \geq \beta$ such that $\left\langle s_{0}, x-x_{0}\right\rangle \notin-$ Int $C$.

PROOF. Define a multifunction $\tilde{F}: X \rightarrow 2^{L(X, Y)}$ for any $x \in X, \tilde{F}(x)=F(x)_{\beta}$. It follows from the $C$-pseudomonotonicity of $F$ that for any $x, y \in X,\langle s, y-x\rangle \notin-I n t C$ for some $s \in \tilde{F}(x)$ implies that $\langle t, y-x\rangle \notin-\operatorname{Int} C$ for some $t \in \tilde{F}(y)$. This implies hat $\tilde{F}$ is $C$-pseudomonotone. Furthermore, the hemicontinuity of $F$ implies the $V$-hemicontinuity of $\tilde{F}$. Since for each $x \in X, F_{x}$ is a compact fuzzy set in $L(X, Y)$, then for each $x \in K, \tilde{F}(x)$ is compact. Condition (2) implies that assumption (2) in Theorem 2 is satisfied for the multifunction $\tilde{F}$. By Theorem 2.1 there exists $x_{0} \in D$ such that for each $x \in D$, there exists $s_{0} \in \tilde{F}\left(x_{0}\right)$ such that $\left\langle s_{0}, x-x_{0}\right\rangle \notin-I n t C$. Hence there exists an $x_{0} \in D$ such that for each $x \in D$, there exists $s_{0} \in L(X, Y)$ with $F_{x_{0}}\left(s_{0}\right) \geq \beta$ such that $\left\langle s_{0}, x-x_{0}\right\rangle \notin-I n t C$.

COROLLARY 3.1. In Theorem 3 , if $D$ is closed, then the coercivity (2) can be replaced by the following without affecting its conclusion:

(2') there exists a nonempty compact subset $K$ of $D$ and a $y_{0} \in K$ such that

$$
\left\langle t, y_{0}-x\right\rangle \in-I n t C \text { for all } x \in D-K \text { and all } t \in L(X, Y) \text { with } F_{y_{0}}(t) \geq \beta \text {. }
$$

For $D=K$, Theorem 3 reduces to the following

COROLLARY 3.2. Let $X$ and $Y$ be Banach space, $C$ a closed pointed and convex cone in $Y$ with Int $C \neq \emptyset, D$ be a nonempty compact and convex subset of $X$ and $F: X \rightarrow \mathcal{F}(L(X, Y))$ a fuzzy mapping such that there exists a real number $\beta \in(0,1]$ such that for each $x \in X,\left(F_{x}\right)_{\beta}$ is a nonempty subset of $L(X, Y)$. Suppose that $F$ is $C$-pseudomonotone and hemicontinuous, and that for each $x \in F, F_{x}$ is a compact fuzzy set in $L(X, Y)$. Then there exists an $x_{0} \in D$ such that for each $x \in D$, there exists an $s_{0} \in L(X, Y)$ with $F_{x_{0}}\left(s_{0}\right) \geq \beta$ such that $\left\langle s_{0}, x-x_{0}\right\rangle \notin-I n t C$.

COROLLARY 3.3 [12]. Let $X$ be a reflexive Banach space and $Y$ a Banach space. Let $D$ be a nonempty, bounded, closed and convex subset of $X$ and $C$ a closed, pointed and convex cone in $Y$ with Int $C \neq \emptyset$. Let $F: X \rightarrow \mathcal{F}(L(X, Y))$ be a fuzzy mapping such that $F$ is $C$-pseudomonotone and hemicontinuous and that for each $x \in X, F_{x}$ is a compact fuzzy set in $L(X, Y)$. Suppose further that there exists a real number $\beta \in(0,1]$ such that for each $x \in X,\left(F_{x}\right)_{\beta}$ is a nonempty subset of $L(X, Y)$. Then there exists an $x_{0} \in D$ such that for each $x \in D$, there exists an $s_{0} \in L(X, Y)$ with $F_{x_{0}}\left(s_{0}\right) \geq \beta$ such that $\left\langle s_{0}, x-x_{0}\right\rangle \notin-I n t C$. 
ACKNOWLEDGEMENT. The first author was supported in part by the Basic Science Research Institute Program, Project No. BSRI-97-1413, the second BSRI-97-1405 and the third BSRI-97-1440.

\section{REFERENCES}

[1] GIANNESSI, F., Theorems of alternative, quadratic programs and complementarity problems, Variational Inequalities and Complementarity Problems (Edited by R.W. Cottle, F. Giannessi and J.L. Lions), John Wiley and Sons, Chichester, England (1980), 151-186.

[2] CHEN, G.Y., Existence of solutions for a vector variational inequality: an extension of the Hartman-Stampacchia theorem, J. Optim. Th. Appl. 74 (3) (1992), 445-456.

[3] CHEN, G.Y. and CHENG, G.M., Vector variational inequality and vector optimization, Lect. Notes in Econ. and Math. System. 285, Springer-Verlag (1987), 408-416.

[4] CHEN, G.Y. and CRAVEN, B.D., Approximate dual and approximate vector variational inequality for multiobjective optimization, J. Austral. Math. Soc. (Series A) 47 (1989), 418-423.

[5] CHEN, G.Y. and CRAVEN, B.D., A vector variational inequality and optimization over an efficient set, Zeitscrift fü Operations Research 3 (1990), 1-12.

[6] CHEN, G.Y. and YANG, X.Q., The vector complementarity problem and its equivalence with the weak minimal element in ordered sets, J. Math. Anal. Appl. 153 (1990), 136-158.

[7] LEE, G.M., KIM, D.S., LEE, B.S. and CHO, S.J., Generalized vector variational inequality and fuzzy extension, Applied Mathematics Letters 6 (6) (1993), 47-51.

[8] CHANG, S.S. and ZHU, Y.G., On variational inequalities for fuzzy mappings, Fuzzy Sets and Systems 32 (1989), 359-367.

[9] LASSONDE, M., On the use of KKM multifunctions in fixed point theory and related topics, J. Math. Anal. Appl. 97 (1983), 151-201.

[10] SHIH, M.H. and TAN, K.K., Generalized quasi-variational inequalities in locally convex topological spaces, J. Math. Anal. Appl. 108 (1985), 333-343.

[11] TAKAHASHI, W., Nonlinear variational inequalities and fixed point theorems, J. Math. Soc. Japan 28 (1976), 168-18i.

[12] YEN, C.L., A minimax inequality and its applications to variational inequalities, Pacific J. Math. 97 (1981), 142-150.

[13] LEE, B.S., LEE, G.M., CHO, S.J. and KIM, D.S., A variational inequality for fuzzy mappings, Proceedings of Fifth International Fuzzy Systems Association World Congress, Seoul (1993), 326329.

[14] KIM, W.K. and TAN, K.K., A variational inequality in non-compact sets and its applications, Bull. Austral. Math. Soc. 46 (1992), 139-148.

[15] PARK, S., Generalizations of Ky Fan's matching theorems and their applications, II, J. Korean Math. Soc. 28 (1991), 275-283.

[16] PARK, S., Some coincidence theorems on acyclic multifunctions and applications to KKM theory, Fixed Point Theory and Applications (Edited by K.-K. Tan), World Scientific, River Edge, NJ (1992), 248-277.

[17] PARK, S., On minimax inequalities on spaces having certain contractible subsets, Bull. Austral. Math. Soc. 47 (1993), 25-40.

[18] COTTLE, R.W. and YAO, J.C., Pseudo-monotone complementarity problems in Hilbert spaces, J. Optim. Th. Appl. 75 (2) (1992), 281-295.

[19] YANG, X.Q., Vector variational inequality and its duality, Nonlinear Anal. TMA 21 (1993), 869877.

[20] WEISS, M.D., Fixed point, separation and induced topologies for fuzzy sets, J. Math. Anal. Appl. 50 (1975), $142-150$. 


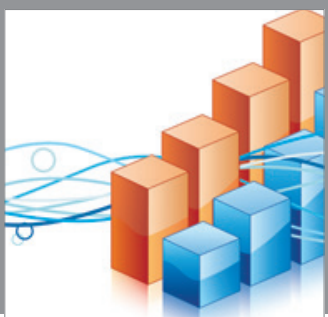

Advances in

Operations Research

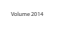

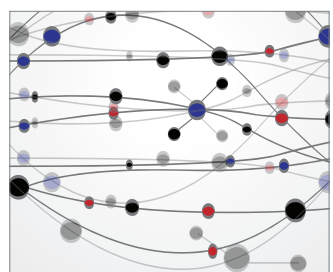

\section{The Scientific} World Journal
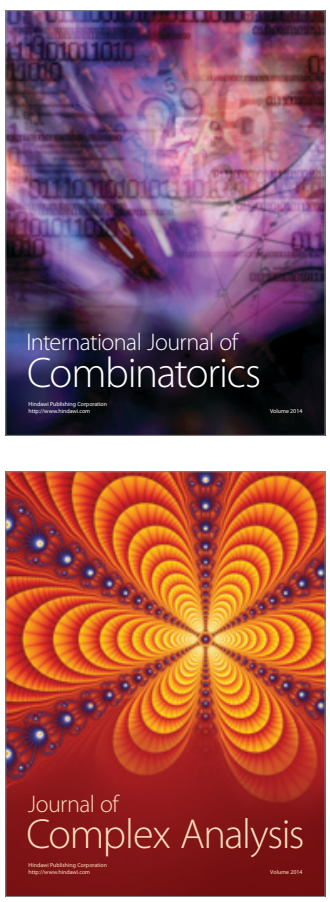

International Journal of

Mathematics and

Mathematical

Sciences
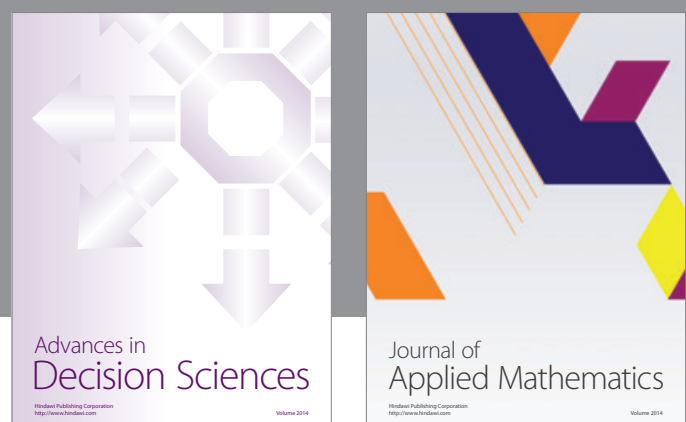

Journal of

Applied Mathematics
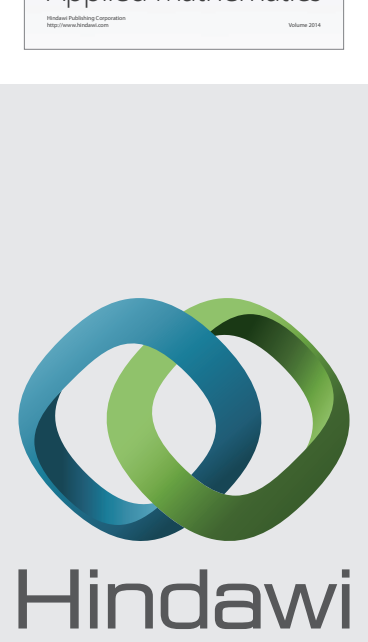

Submit your manuscripts at http://www.hindawi.com
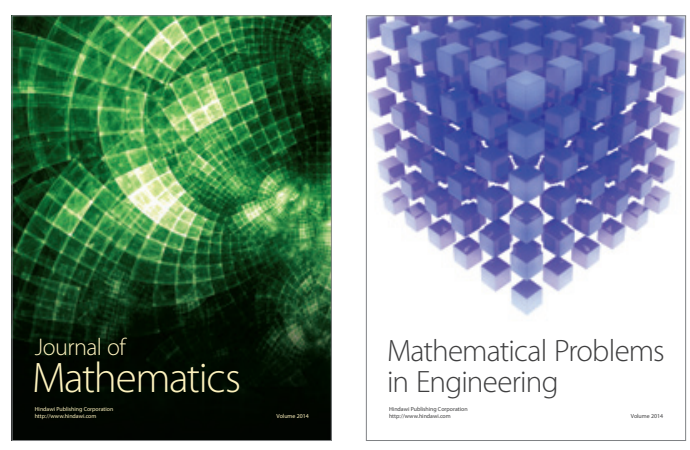

Mathematical Problems in Engineering
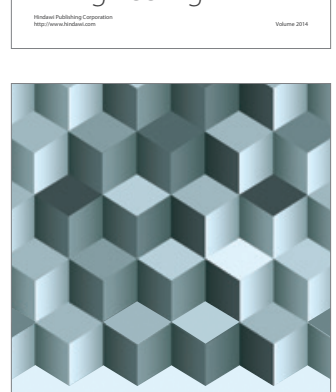

Journal of

Function Spaces
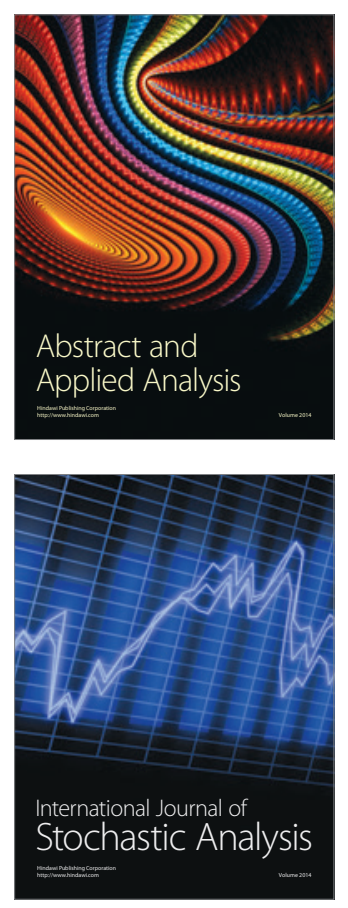

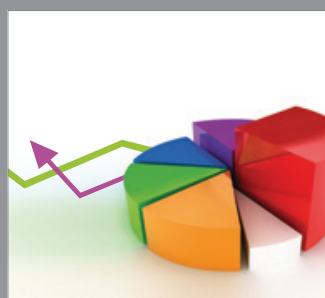

ournal of

Probability and Statistics

Promensencen
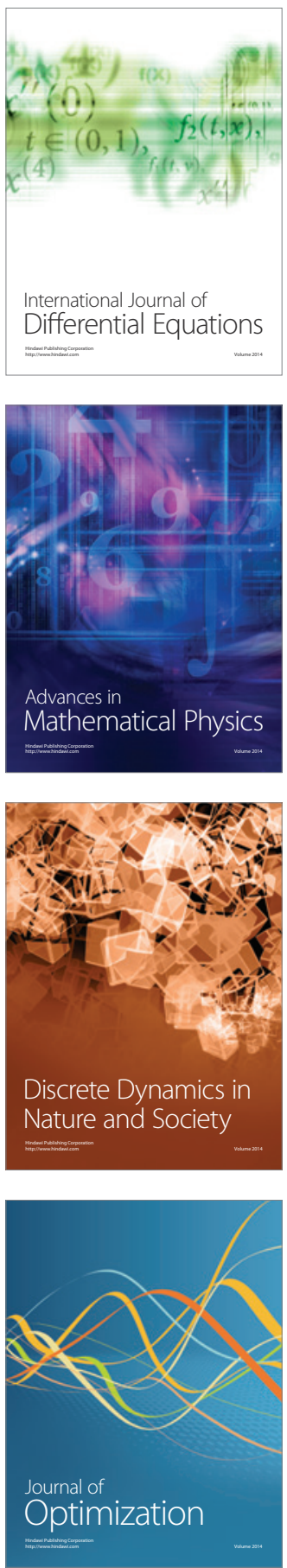